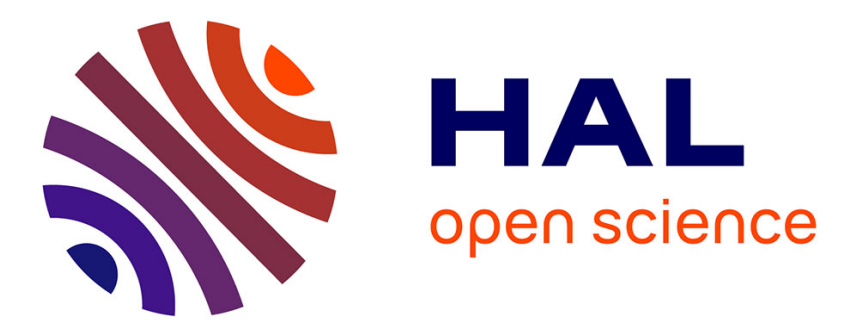

\title{
Entre subversion et nostalgie: l'intertextualité grecque et latine dans The Journal of a Voyage to Lisbon de Henry Fielding
}

Nathalie Bernard

\section{- To cite this version:}

Nathalie Bernard. Entre subversion et nostalgie: l'intertextualité grecque et latine dans The Journal of a Voyage to Lisbon de Henry Fielding. XVII-XVIII Revue de la Société d'études anglo-américaines des XVIIe et XVIIIe siècles , 2005, 60 (1), pp.187-200. 10.3406/xvii.2005.2027 . hal-01386831

\section{HAL Id: hal-01386831 \\ https://hal-amu.archives-ouvertes.fr/hal-01386831}

Submitted on 27 Oct 2016

HAL is a multi-disciplinary open access archive for the deposit and dissemination of scientific research documents, whether they are published or not. The documents may come from teaching and research institutions in France or abroad, or from public or private research centers.
L'archive ouverte pluridisciplinaire HAL, est destinée au dépôt et à la diffusion de documents scientifiques de niveau recherche, publiés ou non, émanant des établissements d'enseignement et de recherche français ou étrangers, des laboratoires publics ou privés. 


\title{
Entre subversion et nostalgie: l'intertextualité grecque et latine dans The Journal of a Voyage to Lisbon de Henry Fielding
} Nathalie Bernard

\begin{abstract}
Fielding's last work, The Journal of a Voyage to Lisbon (1755), claims to be a faithful and accurate report of the author's own travels to Portugal and to have broken free from the extravagant tales traditionally found in fictional travel narratives such as the Odyssey. As always in the context of Fielding's writings, the classical intertexts from the Greek and Latin literatures reinforce the authority of the writer over his readers, as they show off his superior knowledge and great erudition. Yet in the Journal, Fielding undertakes to demythologize those classical texts by translating the supernatural events which they contain into realistic terms, the epic style being used only for the depiction of ordinary or inferior facts and people. Yet, however subversive it may be in its treatment of Greek and Latin intertexts, The Journal of a Voyage to Lisbon is perhaps not totally devoid of nostalgia for the supernatural and the marvellous.
\end{abstract}

\section{Résumé}

The Journal of a Voyage to Lisbon (1755), dernier ouvrage de Henry Fielding, s'ouvre sur une condamnation des invraisemblances qui parsèment les récits de voyage merveilleux tels que l'Odyssée: l'auteur prétend, lui, relater avec exactitude et fidélité les événements de sa traversée. Comme toujours chez Fielding, mais peut-être plus encore dans un contexte désormais marqué par la maladie et l'exil, les intertextes classiques permettent à l'auteur de montrer son érudition et de faire rimer autorité et auctorialité. Dans ce qui restera son ultime récit, Fielding entreprend une démythification des grands textes grecs et latins: il traduit en termes réalistes et rationnels leurs péripéties merveilleuses, tout en réservant l'emploi du style épique aux événements et personnages médiocres rencontrés au cours de son voyage. On tentera cependant de montrer comment The Journal of a Voyage to Lisbon concilie subversion et nostalgie de l'héritage littéraire classique.

\section{Citer ce document / Cite this document :}

Bernard Nathalie. Entre subversion et nostalgie: I'intertextualité grecque et latine dans The Journal of a Voyage to Lisbon de Henry Fielding. In: XVII-XVIII. Revue de la société d'études anglo-américaines des XVIle et XVIIIe siècles. N60, 2005. pp. 187-200.

doi : 10.3406/xvii.2005.2027

http://www.persee.fr/doc/xvii_0291-3798_2005_num_60_1_2027

Document généré le 29/09/2015

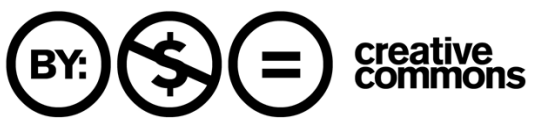




\section{ENTRE SUBVERSION ET NOSTALGIE: L'INTERTEXTUALITÉ GRECQUE ET LATINE DANS THE JOURNAL OF A VOYAGE TO LISBON DE HENRY FIELDING.}

Résumé: The Journal of a Voyage to Lishon (1755), dernier ouvrage de Henry Fielding, s'ouvre sur une condamnation des invraisemblances qui parsèment les récits de voyage merveilleux tels que l'Odyssée: l'auteur prétend, lui, relater avec exactitude et fidélité les événements de sa traversée. Comme toujours chez Fielding, mais peut-être plus encore dans un contexte désormais marqué par la maladie et l'exil, les intertextes classiques permettent à l'auteur de montrer son érudition et de faire rimer autorité et auctorialité. Dans ce qui restera son ultime récit, Fielding entreprend une démythification des grands textes grecs et latins: il traduit en termes réalistes et rationnels leurs péripéties merveilleuses, tout en réservant l'emploi du style épique aux événements et personnages médiocres rencontrés au cours de son voyage. On tentera cependant de montrer comment The Journal of a Voyage to Lisbon concilie subversion et nostalgie de l'héritage littéraire classique.

Abstract: Fielding's last work, The Journal of a Voyage to Lisbon (1755), claims to be a faithful and accurate report of the author's own travels to Portugal and to have broken free from the extravagant tales traditionally found in fictional travel narratives such as the Odyssey. As always in the context of Fielding's writings, the classical intertexts from the Greek and Latin literatures reinforce the authority of the writer over his readers, as they show off his superior knowledge and great erudition. Yet in the Journal, Fielding undertakes to demythologize those classical texts by translating the supernatural events which they contain into realistic terms, the epic style being used only for the depiction of ordinary or inferior facts and people. Yet, however subversive it may be in its treatment of Greek and Latin intertexts, The Journal of a Voyage to Lishon is perhaps not totally devoid of nostalgia for the supernatural and the marvellous. 
The Journal of a Voyage to Lisbon, dernier ouvrage de Henry Fielding, fut publié à titre posthume en 1755. Ce récit relate la traverséc en mer entreprise par l'autcur depuis Londres, où la maladic l'a contraint à abandonner sa fonction officielle de magistrat, jusqu'à Lisbonne, où il espère bénéficier d'un climat plus doux; mais, arrivé à bon port, il meurt bientôt, victime de ses excès de boisson et de bonne chère.

Au moment de la rédaction du Journal, Fielding n'est pas seulement un homme à l'article de la mort et un magistrat privé de ses fonctions officielles; c'est aussi un écrivain meurtri dont le dernier roman, Amelia, paru à la fin de 1751 , a été laminé par la critique. En 1752, dans les pages du Covent Garden Journal, Fielding renonce à l'écriture de fiction devant tant d'hostilité.' The Journal, récit référentiel et historique, illustre ce refus farouche de produire une nouvelle œuvre de fiction romanesque. Les intentions didactiques de Fielding sont clairement indiquées dans la préface: il s'agit pour lui de favoriser la réforme des lois ayant trait au trafic maritime anglais. ${ }^{2}$ L'auteur revendique l'héritage des historiens grecs, tels Xénophon, Thucydide ou encore Hérodote, ${ }^{3}$ et loue à plusieurs reprises la rigueur des écrits de Tite-Live, ${ }^{4}$ Salluste ${ }^{5}$ et Diodore de Sicile. ${ }^{6}$ Le Journal de Fielding se démarque des récits de voyage merveilleux qui trahissent la réalité par leurs excès, et se présente comme une anti-Odyssée, ainsi qu'en témoigne ce fameux passage de la préface:

1. La maladie de Henry Fielding a fait l'objet de plusieurs études: Albert J. Rivero, "Figurations of the Dying: Reading Fielding's The Journal of a Vovage to Lisbon," Journal of English and Germanic Philology 93 (1994): 520-533. Viviès, Jean. "L'auteur à l'article de la mort: le dernier récit de Henry Fielding," $L ' A u t o b i o g r a p h i e$ littéraire en Angleterre (XVII ${ }^{\circ} X X^{\circ}$ siècles): Géographies du soi, ed. Frédéric Regard (Saint-Étienne: P.U.S.E, 2000) 55-70.

2. Henry Fielding, 'A Journey from This World to the Next' and 'The Journal of a Voyage to Lishon' (1755), ed. Ian Bell and Andrew Varney (Oxford: Oxford UP, 1997) 129. Pour ce qui est de la valeur didactique du Journal, voir William J. Burling, "Merit infinitely Short of Service: Fielding's Pleas in The Journal of a Voyage to Lisbon." English Studies 70, 1 (1989): 53-62. L'aspect moralisateur des récits de voyage est étudiée par Jenny Mezciems, "'Tis not to divert the Reader": Moral and Literary Determinants in some Early Travel Narratives," Prose Studies 5, Number 1 (May 1982): 1-20.

3. Fielding 126.

4. Fielding 128.

5. Fielding 128.

6. Fielding 173. 
But, in reality, the Odyssy [sic], the Telemachus, and all of that kind, are to the voyage-writing I here intend, what romance is to true history, the former being the confounder and corrupter of the latter. I am far from supposing that Homer, Hesiod, and the other ancient poets and mythologists, had any settled design to pervert and confuse the records of antiquity; but it is certain they have effected it; and, for my own part, I must confess I should have honoured and loved Homer more had he written a true history of his own times in humble prose, than those noble poems that have so justly collected the praise of all ages. ${ }^{7}$

Je m'appuierai sur la définition de l'intertextualité que donne Gérard Genette dans les premières pages de Palimpsestes. L'intertextualité est, selon lui, "une relation de coprésence entre deux ou plusieurs textes." Celle-ci se manifeste "par la présence effective d'un texte dans un autre," engageant la pratique de la citation, du plagiat ou de la simple allusion. ${ }^{8}$

Dans The Journal of a Voyage to Lisbon, l'intertextualité n'est pas seulement grecque et latine. On y trouve aussi de nombreux intertextes fictionnels britanniques, parmi lesquels la comédie de John Crowne, Sir Courtly Nice, or, It Cannot Be, ou encore Macbeth de Shakespearc. ${ }^{10}$ L'intertextualité référentielle contemporaine n'est pas non plus absente du Journal, ainsi que le montre la mention de l'ouvrage de Montesquieu De l'esprit des lois. " Toutefois, j'aimerais privilégier ici le traitement que The Journal of a Voyage to Lishon fait subir aux récits merveilleux antiques, tels l'Énéide de Virgile, l'Odyssée et l'Iliade d'Homère, ou encore les Métamorphoses d'Ovide, textes souvent cités dans l'ouvrage de Fielding. Il faut rappeler en effet que, si le Journal s'ouvre sur une critique cinglante de l'Odyssée, il s'achève sur un passage tiré des Satires d'Horace. ${ }^{12}$

Je déterminerai tout d'abord la nature du rapport qu'entretient, dans le Journal, l'intertextualité fictionnelle classique avec les notions

7. Fielding 125.

8. Gérard Genette, Palimpsestes: la Littérature au second degré (Paris: Seuil, 1982) 8.

9. Fielding 147.

10. Fielding 163.

11. Fielding 145.

12. Fielding 224. 
d'autorité et d' "auctorialité."13 Il s'agira, ensuite, de voir comment l'évocation de la littérature antique de fiction permet à Fielding de procéder à une littéralisation du merveilleux antique ainsi qu'à un traitement héroï-comique de phénomènes triviaux et de personnages ridicules. Enfin, j'analyserai l'autoportrait de Fielding par l'entremise des allusions intertextuelles classiques qui émaillent son dernier récit.

L'intertextualité classique traduit indéniablement l'érudition de Fielding et confirme son statut de "gentleman" face aux autres individus qui usurpent ce titre, comme le capitaine du navire et son nevcu, tous deux incultes. Évoquant rapidement dans son Journal l'accueillant gentilhomme qui a convié le capitaine à un dîner sur la côte, Fielding le compare à un personnage décrit par Homère, Axylos. ${ }^{14}$ Seul un fin connaisseur des récits antiques peut citer avec aisance et à bon escient le nom d'Axylus (ou Axylos), personnage mincur de l'Iliade, qui n'apparaît que fugacement, pour être d'ailleurs aussitôt mis à mort, au début du livre VI: "Lors Diomède au cri puissant tue Axylos; ce preux fils de Teuthras, dans la bonne Arisbé possédait de grands biens, et les hommes l'aimaient, parce que, demeurant à côté de la route, il faisait grand accueil à tous dans sa maison." 15

Il convient de garder à l'esprit qu'au moment de la rédaction du Journal, la décrépitude physique qui affecte Fielding l'a rendu si dépendant de son entourage qu'il ne peut se déplacer par ses propres moyens. Handicapé, affaibli, soumis à d'humiliantes moqueries, ${ }^{16}$ c'est aussi un romancier désormais boudé par la critique, doublé d'un magistrat que la maladie a éloigné de ses fonctions officielles. Dans ce contexte, l'écriture du Journal en vient presque à remplir une fonction vitale: en l'inscrivant dans une prestigieuse tradition

13. Le terme "auctorialité" est ici employé au sens de "statut de l'auteur," et traduit la notion anglaise d' "authorship" développée par Ian A. Bell dans l'ouvrage Henry Fielding, Authorship and Authority (London: Longman, 1994). Au sujet de l'autorité liée au statut d'auteur de Fielding, voir aussi John Richetti, "The Old Order and the New Novel of the Mid-Eighteenth Century: Narrative Authority in Fielding and Smollett," Eighteenth-Century Fiction 2 (1990) : 183-196.

14. Fielding 200.

15. Homère, L'Iliade, in L'lliade \& l'Odyssée; L'Iliade: traduction, introduction et notes par Robert Flacelière. Odyssée: traduction par Victor Bérard, introduction et notes par Jean Bérard, index par René Langumier (Paris: Gallimard, 1955) 188.

16. Fielding 141. 
littéraire, la culture classique de Fielding renforce son statut d'auteur et permet au voyageur moribond de renouer, ne serait-ce que sur le papier, avec une autorité sociale trop souvent mise à mal par d'irrespectueux inférieurs hiérarchiques. ${ }^{77}$

Parce qu'il traduit l'érudition de l'écrivain, le recours à l'intertextualité antique indique également l'autorité qu'exerce Fielding sur la plupart de ses lecteurs. Le rapport de force entre auteur et lecteur constitue selon Ian $\mathrm{A}$. Bell un phénomène récurrent dans toute l'œuvre de Fielding:

... there is a chronic niggling concern throughout Fielding's writings about whether he can really exert proper authority over his readers, or whether in the end the consumerist ideology of popular publishing means that they have the ultimate power over him. In short, is the author still the figure of authority, or is the customer always right? $^{18}$

La préface du Journal illustre ce comportement auctorial impérieux. Fielding y déclare vouloir préserver tout au long du récit l'alliance de l'utile et de l'agréable. Les lecteurs imprudents qui se piqueraient de critiquer ce principe sont ainsi prévenus: "I have the authority of more than one passage in Horace to allege in my defence." ${ }^{19}$ Dans cette citation, Fielding, à la fois écrivain et homme de loi, manie la référence à Horace comme un indice du bien-fondé de son attitude et la renforce au passage par l'évocation de son pouvoir de magistrat ("to allege in my defence"). Toutefois, il se garde de développer l'allusion à Horace: le lecteur auquel s'adresse Fielding doit savoir que ce poète latin du premier siècle avant J.-C. recommandait de concilier profit et plaisir ("utile dulci"). Le lecteur ignorant, lui, ne sera pas éclairé: l'érudition en matière de littérature classique indique bel et bien ici la supériorité culturelle de l'auteur sur un certain nombre de ses lecteurs.

Cependant, l'utilisation de l'intertextualité grecque et latine dans le Journal de Fielding a aussi pour conséquence de "neutraliser" le

17. Parmi ces derniers figurent le capitaine, qui se veut maître absolu à bord de son navire, Mrs. Francis, l'aubergiste avare et peu amène, ainsi que les douaniers portugais, trop zélés et impolis au goût de Fielding.

18. Bell 238.

19. Fielding 128 . 
pouvoir de la fiction. Il s'agit notamment de réduire les ceuvres imaginaires et mythologiques de l'Antiquité au statut d'œuvres didactiques, de documents instructifs pour le public, et d'en faire ainsi les ancêtres, certes trop farfelus, du Journal lui-même. Notons que, si Fielding prétend dégager les intentions didactiques des récits antiques, il ne mentionne pas les raisons pour lesquelles Virgile ou Ovide auraient alors choisi de recourir à la fiction plutôt qu'à des récits référentiels. Délaissant cette question, il se livre à un étonnant excrcice de "décodage" des textes de fiction classiques, dont les événements extraordinaires deviennent à ses yeux les allégories de situations banales et quotidiennes. Le lecteur assiste tout au long du Journal à ce que l'on pourrait nommer une "littéralisation" de la littérature merveilleuse:

For my own part, however whimsical it may appear, I confess, I have thought the strange story of Circe in the Odyssey [sic], no other than an ingenious allegory; in which Homer intended to convey to his countrymen the same kind of instruction, which w'e intend to communicate to our own in this digression. As teaching the art of war to the Greeks, was the plain design of the Iliad; so was teaching them the art of navigation the no less manifest intention of the Odyssey. ${ }^{20}$

Fielding poursuit cette "littéralisation" lorsqu'il évoque le pouvoir qu'exercent sur les marins les tripots et les maisons closes: ces derniers constituent, en réalité, les charmes, largement répandus dans tous les ports du monde, grâce auxquels Circé captive les hommes. Le Journal traite l'Odyssée comme si le récit attribué à Homère procédait du collage de différentes allégories qu'il s'agissait d'expliciter. Selon le raisonnement de Fielding, le personnage d'Ulysse représente de façon magnifiée le capitaine d'un navire de commerce, tandis que Circé n'est au bout du compte que la traduction dans le style épique d'une vulgaire aubergiste: la transformation des marins en pourceaux n'est que la métaphore de l'enivrement auquel elle les soumet. Dans le but de défendre une interprétation documentaire et didactique des fictions antiques, Fielding use d'arguments qui révèlent son désir profond de déjouer la magie de la fiction, d'en rationaliser les mystères: "thus a key will be found out for unlocking the whole

20. Fielding 194. 
mystery, and forging, at least, some meaning to a story which, at present, appears very strange and absurd."21

C'est la même logique qui prévaut lorsque Fielding propose, à la lumière de son propre voyage en mer, une relecture d'un vers tiré des Métamorphoses d'Ovide: "Omnia Pontus erant, deerant quoque littora Ponto,"22 que Varney traduit ainsi: "everywhere there were waters, and there were no shores to the waters." ${ }^{23}$ En effet, la frêle embarcation qui conduit Fielding depuis le navire vers la côte ne peut atteindre le rivage, car la marée est basse et les voyageurs doivent parcourir une longue étendue boueuse avant de rejoindre la "terre ferme." Cette anecdote donne à l'auteur l'occasion de donner une lecture logique des vers du poète: "an instance which seems to explain those lines of Ovid ... in a less tautological sense, than hath generally been imputed to them." ${ }^{24}$

Cependant cette traduction en termes triviaux des éléments de la fiction classique va de pair avec l'emploi du style héroï-comique pour dépeindre certains personnages ridicules et dérisoires du Journal, comme le capitaine du navire qui conduit Fielding à Lisbonne, ou encore son compère suisse, que l'on voit tous deux occupés à célébrer leurs prouesses ("celebrating their own heroic exploits . . ."). ${ }^{25} \dot{A}$ la rationalisation des récits de fiction épiques correspond, en effet, un phénomène inverse, marqué par le recours à des termes héroïcomique pour qualifier événements banals et personnes médiocres. C'est ce qui se produit dans cette évocation de l'aubergiste Mrs. Francis, où se combinent ironie et parodie du style ćpique: "the good woman had starved us, but from wisely consulting her own dignity, or perhaps, her vanity, to which our hunger was offered as a sacrifice." ${ }^{26}$ Plus loin, l'hôtesse avare et acariâtre adopte les traits de l'une des Furies, Furie malencontreusement installée au Paradis, puisque c'est ainsi que l'île de Wight apparaît à Fielding.

Bien qu'historique, le dernier récit de Fielding, participe donc toujours de la grande entreprise de déstabilisation, de subversion des

21. Fielding 195.

22. Fielding 165 .

23. Fielding 237.

24. Fielding 165.

25. Fielding 204.

26. Fielding 167. 
fondements du récit épique qui a, selon Bakhtine, favorisé l'essor du genre romanesque au XVIIIe siècle, et illustre bien cette citation tirée d'Esthétique et théorie du roman: " 'Le passé absolu' des dieux, demidieux et héros 's'actualise' dans les parodies et surtout dans les travestissements: il est rabaissé, représenté au niveau de l'actualité, dans son cadre habituel, dans le 'bas langage' de son temps." ${ }^{27}$

Enfin, c'est le combat dérisoire que mène le Capitaine contre le vent qui illustre le mieux le style héroï-comique du Journal of a Voyage to Lisbon. Les nombreux caprices du vent qui retardent l'avancée du navire vers Lisbonne évoquent pour Fielding le souvenir d'un épisode relaté dans le premier livre de l'Énéide de Virgile. Junon, sachant que les Troyens menaceront plus tard sa Carthage bienaimée, incite Éole à déchaîner un violent orage sur les bateaux d'Énée si bien que certains des vaisseaux sont détruits, et "la flotte, dispersée": "it was S.S.E. which is that very wind which Juno would have solicited of Æolus, had Æneas been in our latitude bound for Lisbon." ${ }^{28}$

Refusant, contrairement à Fielding, de se soumettre aux forces naturelles qui se jouent de lui, le capitaine fait preuve d'hubris mais se voit contraint d'abandonner ce combat inégal, perdant en quelques minutes le bénéfice de ses efforts: "he hoisted his sails, and, as if his power had been no less absolute over Eolus than it was over Neptune, he forced the wind to blow him on in its own despight."29

Dérisoire Pythie de Delphes, le capitaine multiplie les prophéties qui se révèlent toujours fausses: le vent, personnifić, tient tête à celui qui se croit doté de pouvoirs surhumains ("the wind remained as much his enemy as ever"). ${ }^{30}$ Excédé par tant de déconvenues, le capitaine finit par admettre qu'Éole ne se soumettra pas à son autorité, mais, refusant de n'y voir que l'effet de conditions météorologiques défavorables, il est désormais persuadé qu'une sorcière lui a jeté un sort. C'est tout naturellement Mrs. Francis, l'aubergiste de Ryde, qu'il accuse du maléfice: fâchée de ne pouvoir soutirer plus d'argent à son client Fielding, elle aurait empêché le navire de quitter le port. ${ }^{31}$

27. Mikhaïl Bakhtine, Esthétique et théorie du roman (Paris: Gallimard, 1978) 456.

28. Fielding 218.

29. Fielding 159.

30. Fielding 182.

31 . Fielding 211 . 
Auteur d'un journal qui se veut récit factuel et sérieux, Fielding tourne en dérision la croyance aveugle du capitaine en des phénomènes irrationnels, comme la prophétic ou la sorcellerie: plus encore que l'échec d'un capitaine fruste mais orgueilleux qui se croit, à tort, tout-puissant, c'est bien le recours à l'imaginaire, au merveilleux, que raillent ces portraits cocasses.

Enfin, l'intertextualité classique présente dans The Journal contribue aussi à confronter les figures mythiques des récits antiques à l'autoportrait que trace Fielding dans son dernier récit. Ainsi, aux exploits fabuleux d'Ulysse ou d'Énée correspondent les récents succès publics obtenus par le magistrat, véritable héros moderne. L'introduction du Journal rappelle longuement que Fielding a quitté Londres après avoir remporté une victoire pénible mais décisive sur les bandes de criminels qui sévissaient dans la capitale:

Mean while [sic], amidst all my fatigues and distresses, I had the satisfaction to find my endeavours had been attended with such success, that this hellish society were almost utterly extirpated, and that, instead of reading of murders and street-robberies in the news, almost every morning, there was, in the remaining part of the month of November, and in all December, not only no such thing as a murder, but not even a street-robbery committed. ${ }^{32}$

Le départ de Fielding victorieux, largement décrit dans l'introduction du Journal, est un écho implicite à celui d'Ulysse quittant Troie. En effet, sclon le récit antique, les assaillants grecs sont prêts à abandonner le siège après dix années de combats, lorsque Ulysse conçoit le cheval de bois qui leur ouvre enfin les portes de la cité de Troic. De même, Fielding, grâce à la mise en place de patrouilles de police efficaces, donne-t-il un coup fatal à la pègre londonienne.

Puis, dans l'Odyssée, Ulysse embarque vers un repos bien mérité à Ithaque, sa mère patric: de nombreuses aventures retardent alors son arrivée à bon port. Certes, à l'inverse du héros antique, Fielding ne fait pas route vers son pays natal. Il voit néanmoins, comme Ulysse, sans cesse repoussé son séjour sur des terres plus clémentes: c'est dans le cadre de cette réécriture triviale de l'Odyssée que le Journal

32. Fielding 131 . 
donne à la peu séduisante Mrs. Francis le rôle d'une Circé ou d'une Calypso.

Fielding ne possède malheureusement pas tous les attributs du roi d'Ithaque: ainsi, il n'est pas maître à bord du navire sur lequel il voyage, comme le lui rappelle souvent le comportement despotique du capitaine. De plus, Fielding, lui, ne retournera pas dans sa patrie, auprès des siens. Enfermé dans le corps trop humain d'un mourant, Ulysse affaibli, l'auteur n'en est pas moins un homme courageux dont la résistance à la souffrance physique définit un hérö̈sme à échelle humaine. C'est avec une fierté teintée de dérision que Fielding évoque la patience héroïque ("heroic constancy") ${ }^{33}$ avec laquelle il endure à plusieurs reprises au cours du Journal de douloureuses opérations destinées à ponctionner à l'aide d'une canule les liquides qui engorgent ses tissus abdominaux.

C'est sans doute cet héroïsme du quotidien que Fielding s'applique à mesurer dans le Journal: il évalue la distance qui sépare les exploits spectaculaires des héros épiques des actes apparemment dérisoires, mais parfois admirables, dont sont capables les hommes. Il ne cherche cependant pas à brosser de lui-même un portrait idéalisé. Ainsi qu'il le confesse dans l'introduction du Journal, sa vanité l'incite quelquefois à se hisser au rang des héros qui ont sacrifié leur vie au salut de la "Res Publica," tel un Curtius se jetant dans les abîmes pour préserver Rome. Il reconnaît bien vite l'outrance de cette comparaison qu'il ramène à des proportions triviales, d'ordre domestique: "And tho' I disclaim all pretence to that Spartan or Roman patriotism, which loved the public so well that it was always ready to become a voluntary sacrifice to the public good, I do solemnly declare I have that love for my family." ${ }^{34}$ Toutefois, il déclare qu'il a su trouver le courage de lutter contre la pègre londonienne malgré sa maladie grâce à un hémistiche de l'Énéide exhortant les hommes à affronter l'adversité: "superanda omnis fortuna ferendo est." ${ }^{35}$

Les références aux intertextes épiques continuent de hanter le Journal. Ainsi, dans les dernières lignes du récit apparaît une citation

35. Fielding 182. 
issue de l'Énéide ("Egressi optata Troes potiuntur aerena"). ${ }^{36}$ S'il ne prétend plus au titre de nouvel Ulysse, héros épique par excellence, $\mathrm{du}$ moins peut-être Fielding partage-t-il quelques-unes des caractéristiques d'Énée, le Troyen vaincu qui vogue vers une nouvelle patrie, guidé par son esprit civique.

La description de Lisbonne complète l'autoportrait que livre Fielding dans le Journal et évoque les villes mythiques des récits antiques. Selon la tradition, relayée Les Lusiades, épopée composée par Luís de Camões au XVIe siècle, Lisbonne aurait été fondée par Ulysse. $^{37}$ Le mythe fut, d'ailleurs, conforté par la ressemblance phonétique entre le nom du héros grec et l'ancien toponyme de la ville: Olisipo. Ville d'exception que Maria de Fatima Antunes Rambaud décrit comme "la dernière marche avant l'Atlantide engloutie, ${ }^{n 38}$ Lisbonne se vit tout naturellement prêter des origines mythiques. Mais le sort que réserve Fielding à cette ville dans son Journal est tout autre. Il connaissait peut-être la légende de sa fondation, mais son récit n'en fait pas mention et présente plutôt la capitale du Portugal comme un reflet dégradé de Rome. Selon lui, Lisbonne est un leurre qui ne peut que décevoir les nouveaux venus. Comme la cité antique, elle est construite sur sept collines, mais un malencontreux effet d'optique écrase les reliefs de la ville, si bien que le voyageur qui la contemple depuis le port ne peut se rendre compte de cette flatteuse coïncidence. ${ }^{39} \mathrm{Et}$, si Lisbonne cependant paraît belle à qui l'observe depuis les flots, cette promesse de beauté s'avère une illusion de plus, un trompe-l'oeil dont le regard décèle vite l'artifice:

As the houses, convents, churches, \&c. are large, and all built with white stone, they look very beautiful at a distance, but as you approach nearer, and find them to want every kind of ornament, all idea of beauty vanishes at once. ${ }^{40}$

36. Fielding 224. Traduction p. 241: "Having disembarked the Trojans took possession of the longed-for sands."

37. Luís De Camões, Les Lusiades, traduction, introduction et notes de Roger Bismut (1954; 1992; Paris: Laffont, 1996).

38. Maria de Fatima Antunes Rambaud, "Olisipona-Lisbonne: le tribut d'Ulysse," in Le Rivage des mythes. Une Géocritique méditerranéenne. le lieu et son mythe, ed. Bertrand Westphal, Collection Espaces Humains (Limoges: Presses Universitaires de Limoges, 2001) 367-377.

39. Fielding 223.

40. Fielding 223. 
Pour finir, Lisbonne n'est pas, selon Fielding, la digne héritière de Rome, mais bien plutôt une nouvelle Troie. L'auteur déclare, en effet, que si les lois qui s'appliquent aux visiteurs récemment arrivés au Portugal sont si sévères, c'est sans doute pour éviter que ne se répète le piège du cheval de Troie:

. . . they were willing to guard against the bare possibility of surprize, of the success of which bare possibility the Trojan horse will remain for ever on record, as a great and memorable example. Now the Portuguese have no walls to secure them, and a vessel of two or three hundred tuns will contain a much larger body of troops than could be concealed in that famous machine, tho' Virgil tells us (somewhat hyperbolically, I believe) that it was as big as a mountain. ${ }^{41}$

Là encore, le mythe est transposé à la période contemporaine, le cheval monumental devient simple navire. Le charme de la référence classique est comme annulé, et le lecteur du Journal assiste, en quelque sorte, à un tour de magie à rebours. Quant à la réserve émise par Fielding au sujet de la taille du cheval de Troie dans la citation cidessus, elle pourra sembler révélatrice de la démythification opérée dans le Journal. En effet, Fielding s'attache constamment à réduire les situations nobles à des phénomènes banals, sans grandeur ni sublime, réservant les hyperboles à la description des personnes et événements médiocres auxquels elles confèrent un style héroï-comique.

Fielding rompt officiellement (mais seulement en apparence) ${ }^{42}$ avec l'écriture de fiction en signant The Journal of a Voyage to Lisbon: prenant explicitement le contre-pied des récits merveilleux de l'Antiquité, le Journal prétend relater avec exactitude et fidélité les faits réels, dans le but affiché d'agir sur eux en incitant le pouvoir politique à une réforme du trafic maritime anglais. Tout d'abord, le recours aux intertextes grecs et latins permet au magistrat malade, doublé d'un romancier humilié par la critique, de manifester son érudition et de démontrer sa supériorité culturelle sur les membres de l'équipage et sur une partie de son lectorat.

41. Fielding 224.

42. Voir l'introduction de Ian A. Bell dans son édition du Journal, et plus particulièrement les pages xix à xxix (Oxford: Oxford UP, 1997). 
Toutefois, comme l'a annoncé l'introduction du Journal, Fielding n'est pas seulement l'héritier des auteurs classiques: condamnant les invraisemblances des récits de fiction antiques, il entreprend de traduire en termes réalistes et rationnels les événements magiques ou mystérieux qui émaillent les textes grecs ou latins. Cette relecture démythifiée de quelques grands récits épiques de l'Antiquité recourt au style héroï-comique pour dépeindre les événements et les personnages médiocres rencontrés au fil du voyage. Ce phénomène binaire consiste à remettre en question la trivialité du monde en y juxtaposant la dignité de l'épique, et à mettre en question les prétentions sublimes de l'épique en y opposant les désagréments de la vie.

Cet ultime récit est pour Fielding l'occasion d'un autoportrait changeant où il adopte tour à tour les traits d'Ulysse et du Troyen Énée. En convoquant ces intertextes antiques, le Journal traduit les désillusions et les interrogations d'un homme qui se sait condamné par la maladie et évalue l'héroïsme épique à l'aune des tourments bien réels dont il est la victime à la fois pathétique et valeureuse. Si la vie se révèle plus triviale que dans les récits mythiques, l'héroïsme n'en est peut-être pas moins admirable pour ĉtre plus humain. La comparaison entre vic et texte se poursuit tout au long du Journal jusqu'à la citation finale extraite des Satires d'Horace, qui marque la concomitance entrc récit ct voyage. ${ }^{43}$ Le parcours au fil des pages du livre prend fin au moment où s'achève le trajet sur les eaux: "his Finis chartaeq; viaeq;.[sic]"). ${ }^{44}$

Certes, Fielding mesure désormais l'écart qui sépare la vie d'un récit épique: il s'en amuse et s'en indigne tout à la fois. Et peut-être également, malgré lui, s'en émerveille-t-il encore. Comme l'indique la citation finale où sa voix se confond avec celle, satirique, d'Horace, il semble que, jusqu'au terme du voyage, point final du récit et presque de sa vie, Fielding demeure malgré lui sous le charme des récits classiques qui incarnent à ses yeux le pouvoir auctorial dans toute sa démesure. Les textes classiques ne constituent pas seulement des vecteurs d'autorité culturelle et, par là, sociale: ils consacrent aussi le

43. Horace, Satires (Sermonum Libri) ed. François Villeneuve, Collection des Universités de France (Paris: Belles Lettres, 1962).

44. Fielding 224. Traduction en note p. 241 : "this is the end of the story and of the journey." 
triomphe de l'imagination sur les faits réels. Coupable de soustraire l'esprit aux tracas quotidiens, mais par là même désirable, accusée de trahir l'héroïsme humain mais lui rendant hommage par son sublime, l'intertextualité classique merveilleuse est, certes, pour Fielding objet de critique, de dérision, mais toujours d'attention. Le Journal se clôt, ainsi, entre subversion et nostalgie.

\section{Nathalic BERNARD}

Université de Provence-Aix-Marseille I 